\title{
APPLICATION ON LOCAL DISCRETE EXPANSION
}

\author{
M.E. ABD EL-MONSEF, A.M. KOZAE and A.A. ABO KHADRA \\ Department of Mathematics, Faculty of Science \\ Tanta University, Tanta, EGYPT
}

(Received June 2, 1992 and in revised form September 14, 1995)

\begin{abstract}
The process of changing a topology by some types of its local discrete expansion preserves $s$-closeness, $S$-closeness, semi-compactness, semi- $T_{\imath}$, semi- $R_{\imath}, i \in\{0,1,2\}$, and extremely disconnectness Via some other forms of such above replacements one can have topologies which satisfy separation axioms the original topology does not have
\end{abstract}

KEY WORDS AND PHRASES: Near open sets, local discrete expansion, extremely disconnected, semi-compact, $s$-closed, $S$-closed, semi- $T_{\imath}$, semi- $R_{\imath}$, and cid spaces

1991 AMS SUBJECT CLASSIFICATION CODES: 54A10, 54D10, 54D30, 54G20

\section{INTRODUCTION}

Throughout the present paper $(X, \tau)$ is a topological space (or simply a space $X$ ) on which no separation axioms are assumed unless explicitly stated. For any $B \subset X, c l_{\tau} B$ (resp int $_{\tau} B$ ) denotes the closure (resp interior) of $B$ A subset $B$ is said to be regular open (resp regular closed) if $B=$ int $_{\tau}$ $\left(c l_{\tau}(B)\right)\left(\right.$ resp $B=c l_{\tau}\left(\right.$ int $\left._{\tau}(B)\right)$ ) A subset $B$ of a space $X$ is said to be $\tau$-semi open [12] (resp $\tau$ regular semi-open [2]) if there exists a $\tau$-open (resp. $\tau$-regular open) set $U$ satisfying $U \subset B \subset c l_{\tau} U \quad B$ is $\tau$-semi-closed [3] if the set $X-B$ is $\tau$-semi-open. The family of all regular open (resp regular semiopen, semi-open) sets in $X$ is denoted by $R O(X, \tau)$ (resp $R S O(X, \tau), S O(X, \tau)$ ) The union (resp intersection) of all $\tau$-semi-open (resp $\tau$-semi-closed) sets contained in $B$ (resp containing $B$ ) is called the $\tau$-semi-interior [3] (resp $\tau$-semi-closure [3]) of $B$, and it is denoted as $s$-int ${ }_{\tau} B$ (resp $s-c l_{\tau} B$ ) A space $X$ is said to be extremely disconnected (denoted by E.D) if for every open set $U$ of $X, c l_{\tau} U$ is open in $\tau$ The concept of local discrete expansion of a topology was first introduced by S P Young in 1977 [17], "Let $(X, \tau)$ be a topological space and $A$ be any subset of $X$ The topology $\tau[A]=\{U-H: U \in \tau, H \subset A\}$ is called the local discrete expansion of $\tau$ by $A$ A space $X$ is semi$T_{2}$ [13] (resp semi- $T_{2}^{\prime}$ [1]) iff for $x, y \in X, x \neq y$ there exist $U$ and $V \in S O(X, \tau), x \in U$ and $y \in V$ such that $U \cap V=\phi$ (resp $\left.c l_{\tau} U \cap c l_{\tau} V=\phi\right)$. Semi- $T_{0}$ and semi- $T_{1}$ were introduced to topological spaces [13] by replacing the word "open" by "semi-open" in the definitions of $T_{0}$ and $T_{1}$ respectively A space $X$ is semi- $R_{0}[6]$ iff for each semi-open set $U$ and $x \in U, s-c l_{\tau}\{x\} \subset U$ A space $X$ is semi- $R_{1}$ [6] iff for $x, y \in X$ such that $s-c l_{\tau}\{x\} \neq s-c l_{\tau}\{y\}$ there exist disjoint semi-open sets $U$ and $V$ such that $s-c l_{\tau}\{x\} \subset U$, and $s-c l_{\tau}\{y\} \subset V$. A space $X$ is called cid [15] if every countable infinite subspace of $X$ is discrete. A space $X$ is semi-compact [7] (resp s-closed [5], $S$-closed [16]) if for every cover $\left\{V_{2}: i \in I\right\}$ of $X$ by semi-open sets of $X$, there exists a finite subset $I_{0}$ of $I$ such that $X=\cup\left\{V_{\imath}: i \in I_{0}\right\}$ (resp $\left.\left.X=\cup \operatorname{scl}\left(V_{\imath}\right): i \in I_{0}\right\}, X=\cup \operatorname{cl}\left(V_{\imath}\right): i \in I_{0}\right\}$ ).

REMARK 1.1. For a subset $A$ of a space $(X, \tau)$ we say that $A$ satisfies condition $\left(C_{1}\right)$ if $A \cup U=\phi$, for every $U \in \tau-\{X\}$.

Listed below are theorems that will be utilized in this paper

THEOREM 1.1 [14] If $\tau$ and $\tau^{\prime}$ are two topologies on $X$ such that $\tau \subset \tau^{\prime}$, then $R O(X, \tau)=$ $R O\left(X, \tau^{\prime}\right)$ iff $c l_{\tau} G=c l_{\tau^{\prime}} G$ for every $G \in \tau^{\prime}$ [equivalent iff int ${ }_{\tau} F=$ int $_{\tau^{\prime}} F$, for every $F \in{\tau^{\prime c}}^{c}$ ]

THEOREM 1.2 [11] If $X$ is a space, and $A \subset X$ satisfying $\left(C_{1}\right)$ Then, $c l_{\tau \mid A]} G=c l_{+} G$, for every $G \in \tau[A]$ 
THEOREM 1.3 [4] If $X$ is a space, and $A \in S O(X, \tau)$ such that $A \subset B \subset c l, A$ Then, $B \in S O(X, \tau)$

THEOREM 1.4 [10] If $X$ is a space, and $B \subset X$, then $s-c l, B=B \cup$ int $_{1} c l_{7} B$

THEOREM 1.5 [8] A space $X$ is E D iff for every pair $U$ and $V$ of disjoint $\tau$-open sets, we have $c l_{7} U \cap c l_{\tau} V=\phi$

THEOREM 1.6 [5] A space $X$ is $s$-closed iff every cover of $X$ by regular semi-open sets has a finite subcover

THEOREM 1.7 [15] (a) A space $X$ is cid if every countable infinite subset is closed

(b) Any infinite cid space is $T_{1}$

THEOREM 1.8 [17] Let $A$ be any subset of $X$ Then $(A, \tau[A] \cap A)$ is discrete

THEOREM 1.9 [17] Let $A$ be a closed subset of $X$ Then $(A, \tau \cap A)$ is a discrete subspace of $X$ iff $\tau=\tau[A]$

THEOREM 1.10 [9] Let $X$ be a $T_{1}$-space Then $X$ is cid iff countable subsets have no limits points

\section{ON LOCAL DISCRETE EXPANSION}

THEOREM 2.1. If $(X, \tau)$ is a space and $A \subset X$, then

(i) $S O(X, \tau[A]) \subset\{B-H: B \in S O(X, \tau), H \subset A\}$

(ii) If $A$ satisfying $\left(C_{1}\right)$, then the inclusion symbol in (i) is replaced by equality sign

PROOF. (i) Let $W \in S O(X, \tau[A])$, then there exists $V \in \tau[A]$ such that $V \subset W \subset c l_{\tau[A]} V$ Then $\left(U-H_{1}\right) \subset W \subset c l_{\tau[A]}\left(U-H_{1}\right)$, where $U \in \tau, H_{1} \subset A$ Put $H_{2}=U \cap H_{1}$, then $H_{2} \subset A$, and $\left(U-H_{1}\right) \cup H_{2} \subset W \cup H_{2} \subset c l_{\tau[A]}\left(U-H_{1}\right) \cup H_{2} \quad$ Then $U \subset W \cup H_{2} \subset c l_{\tau[A]} U \subset c l_{\tau} U$, and $\left(W \cup H_{2}\right) \in S O(X, \tau) \quad$ Put $B=W \cup H_{2}$, and $H=H_{1}-W \subset A$ Then $B-H=$ $W \cup\left(U \cap H_{1}\right)-\left(H_{1}-W\right)=W$.

(ii) By Theorem 1.2, the proof is obvious

REMARK 2.1. From Theorem 2.1, it is easy to prove that, for any $A \subset X$

$S O(X, \tau) \subset S O(X, \tau[A])$

THEOREM 2.2. If $(X, \tau)$ is a space, and $A \subset X$ satisfying $\left(C_{1}\right)$ Then

(i) $S O(X, \tau)=S O(X, \tau[A])$.

(ii) $R S O(X, \tau)=R S O(X, \tau[A])$.

PROOF. In general $S O(X, \tau) \subset S O(X, \tau[A])$. To prove the converse, let $W \in S O(X, \tau[A])$, then there exists $V \in \tau[A]$ satisfying $V \subset W \subset c l_{\tau[A]} V$. Then $(U-H) \subset W \subset \operatorname{cl}_{\tau[A]}(U-H)$, $U \in \tau, H \subset A$. There are two cases.

(a) $U \neq X$, then $U-H=U$ Since $c l_{\tau[A]} U=c l_{\tau} U$, then $W \in S O(X, \tau)$.

(b) $U=X$, then $(X-H) \subset W \subset c l_{\tau[A]}(X-H) \subset c l_{\tau}(X-H)$. Since $A \cap U=\phi$, then $c l_{\tau} A \subset(X-U)$, and $c l_{\tau} A \cap U=\phi$, implies to $c l_{\tau} H \cap U=\phi$, for each $U \in \tau-\{X\}$ Hence $U \not \subset c l_{\tau} H$, and $\operatorname{int}_{\tau} c l_{\tau} H=\phi$, and $H$ is a $\tau$-semi-closed set Thus $(X-H) \in S O(X, \tau)$ From Theorem $13, W \in S O(X, \tau)$

(ii) By Theorems 1.1 and 12 , the proof is obvious

COROLLARY 2.1. If $X$ is a space, and $A \subset X$ satisfying $\left(C_{1}\right)$ Then

(i) $(X, \tau)$ is semi- $T_{\imath}$ iff $(X, \tau[A])$ is semi- $T_{\imath}(i \in\{0,1,2\})$

(ii) If $(X, \tau)$ is semi- $T_{2}^{\prime}$, then $(X, \tau[A])$ is semi- $T_{2}^{\prime}$.

(iii) If $(X, \tau)$ is semi- $R_{\imath}$, then $(X, \tau[A])$ is semi- $R_{\imath}(i \in\{0,1\})$

PROOF. By Theorem (2 2), the proof is obvious

THEOREM 2.3. If $X$ is a space, and $A \subset X$ satisfying $\left(C_{1}\right)$. Then $s-c l_{\tau[A]} G=s-c l_{\tau} G$, for every $G \in \tau[A]$

PROOF. Let $G \in \tau[A]$, then $s-c l_{\tau[\mathrm{A}]} G=G \cup \operatorname{int}_{\tau[A]} c l_{\tau[A]} G=G \cup \operatorname{int}_{\tau} c l_{\tau[A]} G=G \cup \operatorname{int}_{\tau} c l_{\tau} G=$ $s-c l_{\tau} G$ [by Theorems 11,12 and 14$]$ 
THEOREM 2.4. If $X$ is a space, and $A \subset X$ satisfying $\left(C_{1}\right)$. Then $(X, \tau)$ is E.D. iff $(X, \tau[A])$ is E D PROOF. Let $(X, \tau)$ be E.D., $W \in \tau[A]$ Then $W=U-H, U \in \tau, H \subset A$.

But $c l_{\tau[A]}(U-H)=c l_{\tau[A]} U=c l_{\tau} U$, and $c l_{\tau} U \in \tau$. Thus $c l_{\tau[A]} W \in \tau[A]$, and $(X, \tau[A])$ is E.D Conversely, let $(X, \tau[A])$ be E.D., and $U, V \in \tau$ such that $c l_{\tau} U \cap c l_{\tau} V \neq \phi$. By Theorem 1.2, $c l_{\tau[A]} U \cap c l_{\tau[A]} V \neq \phi$, then $U \cap V \neq \phi$ [by Theorem 1.5]. Hence $(X, \tau)$ is E.D.

THEOREM 2.5. If $X$ is a space, and $A \subset X$ satisfying $\left(C_{1}\right)$. Then $(X, \tau)$ is semi-compact (resp sclosed) iff $(X, \tau[A])$ is semi-compact (resp. $s$-closed).

PROOF. By Theorem 2.2, the proof is obvious.

THEOREM 2.6. If $X$ is a space, and $A \subset X$, and $(X, \tau[A])$ is $S$-closed (resp. s-closed), then $(X, \tau)$ is $S$-closed (resp. $s$-closed).

PROOF. Since $S O(X, \tau) \subset S O(X, \tau[A])$, the proof is obvious.

\section{3. $L-T_{i}$ AND $Q-L-T_{i}$ SPACES}

Let $R$ be a topological property which is preserved under expansions

DEFINITION 3.1. A topological space $(X, \tau)$ is called $L-R$ if there exists a subset $S \subset X$ and $S \neq X$, such that $(X, \tau[S])$ has $R$.

PROPOSITION 3.1. If $\tau \subset \tau^{\prime}$, then for any $S \subset X, \tau[S] \subset \tau^{\prime}[S]$.

REMARK 3.1. If $\tau \subset \tau^{\prime}$ and $\tau$ is $L-R$, then $\tau^{\prime}$ is also $L-R$, i e. any expansion of $L-R$ topology on $X$ is also $L-R$.

DEFINITION 3.2. Let $i=1,2,2.5$ and $j=0,1,2,2.5$. We say that $(X, \tau)$ is $Q-L-T_{\imath}$, if it is $L-T_{i}$ and $T_{j}$ where $j<i$.

Now we are going to show that some of the properties $L-T_{\imath}$ and $Q-L-T_{\imath}$ are satisfied for some spaces but not for some other spaces.

PROPOSITION 3.2. For a space $X$, the following diagram is easily obtained.

$$
T_{2 \frac{1}{2}} \Rightarrow Q-L-T_{2 \frac{1}{2}} \Rightarrow T_{2} \Rightarrow Q-L-T_{2} \Rightarrow T_{1} \Rightarrow Q-L-T_{1} \Rightarrow T_{0} .
$$

EXAMPLE 3.1. Let $X=\{a, b, c, d\}$ and $\tau=\{\phi, X,\{a, b\},\{c, d\}\}$ is not $T_{0}$ if $A=\{a, c\}$, then $\tau[A]=\{\phi, X,\{b\},\{d\},\{b, d\},\{a, b\},\{c, d\},\{b, c, d\},\{a, b, d\}\}$ is $T_{0}$. This example is $Q-L-T_{0}$.

The following is an example of a $Q-L-T_{2.5}$ but not $T_{2.5}$.

EXAMPLE 3.2. Let $X=N \times Z \cup\{(-1,0),(-1,-1)\}$ where $N$ is the natural numbers and $Z$ the integers. The topology has as its base sets of the following forms:

$$
\begin{aligned}
& \{(m, n)\}, \quad n \neq 0, \quad m \neq-1 \\
U_{n}((a, 0))= & \{(a, 0)\} \cup\{(a, m)|| m \mid \geq n\}, \quad n \in N \\
U_{n}((-1,1))= & \{(-1,1)\} \cup\{(a, m) \mid a \geq n, m>0\}, \quad n \in N \\
U_{n}((-1,-1))= & \{(-1,-1)\} \cup\{(a, m) \mid a \geq n, m<0\}, \quad n \in N .
\end{aligned}
$$

This space is $T_{2}$ but not $T_{2.5}$ as $(-1,1)$ and $(-1,-1)$ do not have disjoint closed neighborhoods. Choosing $A=N \times(Z-\{0\})$, the discrete expansion is the discrete topology and thus $T_{2}$.

EXAMPLE 3.3. Let $X=\{a, b, c, d\}$ and $\tau=\{\phi, X,\{b\},\{d\},\{b, d\},\{a, b\},\{c, d\},\{a, b, d\}$, $\{b, c, d\}\}$, then $\tau[A]=$ Discrete. This example is $Q-L-T_{1}$ but not $T_{1}$ and is an example of a space which is not $Q-L-T_{2}$.

EXAMPLE 3.4. Let $X=\{a, b, c\}$ and $\tau=\{\phi, X,\{a, b\}\}$. If $A=\{a, b\}$, then $\tau[A]=$ Discrete This example is not $Q-L-T_{1}$.

The excluded point topology on an infinite set $X$ is the family consisting of $\phi$ and all subsets of $X$ not containing a point $p$ of $X$.

EXAMPLE 3.5. The excluded point topology is $L-T_{1}$ and not $L-T_{2}$ (also is an example of $Q-L-T_{1}$ but not $\left.T_{1}\right)$.

PROOF. If $X$ is an infinite set and $p$ is the excluded point and $A \subset X$, then:

(i) If $p \notin A$, we have $\tau[A]=\tau \cup\{X-B: B \subset A\}$. Thus $\tau[A]$ is $T_{1}$ but not $T_{2}$. 
(ii) If $p \in A$, then $A$ is closed, and there are two cases

(a) If $B \subset A, p \in B$ in this case any open set in $\tau[A]$ is open in $\tau$, i e $\tau=\tau[A]$

(b) If $B \subset A, p \notin B$ as (i) Thus $\tau[A]=\tau \cup\{X-B \cdot B \subset A\}$

EXAMPLE 3.6. Let $X=[0,1\}$ and $\tau=\{\phi, X, A \subset X \cdot X-A$ is finite $\}$ If we take $S=(0,1]$, then $\tau[S]$ is the Discrete space This example is $Q-L-T_{2}$ but not $T_{2}$

THEOREM 3.1. $(X, \tau)$ is cid space iff $\tau=\tau[A]$ whenever $A$ is a countable infinite subset of $X$

PROOF. We assume that $(X, \tau)$ is cid, then $A$ is closed and discrete subspace By Theorem 19 we have that $\tau=\tau[A]$ Conversely we assume that $\tau=\tau[A]$ By Theorem 18, we have that $(A, \tau \cap A)$ is a discrete subspace of $X$ and $(X, \tau)$ is cid space

THEOREM 3.2. Every space $(X, \tau)$ is $L-T_{0}$

PROOF. Assume that $x_{0} \in X \quad$ We aim to prove that $\tau\left[X-\left\{x_{0}\right\}\right]$ is $T_{0} \quad$ For this purpose let $x, y \in X, x \neq y$, if $U \in \tau$ is an open set containing $x$, then $U-\{y\}$ is an open set in $\tau\left[X-\left\{x_{0}\right\}\right]$ and not containing $y$ If $x_{0}=x$, then $X-\{y\}$ is an open in $\tau\left[X-\left\{x_{0}\right\}\right]$ and not containing $y$ This completes the proof

The following example illustrates a $Q-L-T_{2}$ space but not $T_{2}$

EXAMPLE 3.7. (Countable complement topology [16]) If $X$ is an uncountable set, we define the topology of countable complements on $X$ by declaring open all sets whose complements are countable, together with $\phi$ and $X \quad(X, \tau)$ is $T_{1}$ but not $T_{2} \quad$ Let $A \subset X$ such that $X-A$ is countable For $x_{0} \in X-A, A \cup\left\{x_{0}\right\}$ is $\tau$-open, and so $\left(A \cup\left\{x_{0}\right\}\right)-A=\left\{x_{0}\right\} \in \tau[A] \quad$ For $x_{0} \in A, A$ is $\tau$-open, which means that $A-\left(A-\left\{x_{0}\right\}\right)=\left\{x_{0}\right\}$ is $\tau[A]$-open Thus $\tau[A]$ is discrete and consequently $T_{2}$

UNSOLVED PROBLEM. If $(X, \tau)$ is a space which does not have a property $P$, what are the properties of the subset $A$ that make $(X, \tau[A])$ have $P$ (for $P=$ fixed property)

ACKNOWLEDGMENT. We would like to thank the referee for valuable comments and suggestions, especially Example 32 , Example 37 and Theorem 32

\section{REFERENCES}

[1] ABD EL-MONSEF, M E, Studies on some pretopological concepts, Ph.D Thesis, Tanta University (1980)

[2] CAMERON, D E, Properties of $S$-closed spaces, Proc. Amer. Math. Soc. 72(3) (1978), 581-585

[3] CROSSLEY, S G and HILDEBRAND, S K, Semi-closure, Texas J. Sci. 22 (1971), 99-112

[4] CROSSLEY, S G and HILDEBRAND, S K, Semi-topological properties, Fund. Math. 74 (1972), 233-253

[5] DIMAIO, G and NOIRI, T., On s-closed spaces, Indian J. Pure Appl. Math. 18(3) (1987). 226-233

[6] DORSETT, C H, Semi- $T_{2}$, semi- $R_{1}$ and semi- $R_{0}$ topological spaces, Ann. Soc. Scl. Bruxelles ser. I, 92 (1978), 143-159, M R 80 a 54026.

[7] DORSETT, C H., Semi-convergence and semi-compactness, Indian J. M.M. XIX(I) (1981)

[8] ENGELKING, R., General Topology, Warszawa, 1977.

[9] GANSTER, M, REILLY, I.L. and VAMANAMURTHY, MK, On spaces whose denumerable subspaces are discrete, Math. Bechnk 39 (1987), 283-292.

[10] JANKOVIC, D S. and REILLY, I L, On semi separation properties, Indıan J. Pure Appl. Math. 16(9) (1985), 957-964

[11] LASHIN, E F , A study on extensions of topologies, Ph D Thesis, Tanta University (1988)

[12] LEVINE, N, Semi-open sets and semi continuity in topological spaces, Amer. Math. Monthly 70 (1963), 36-41

[13] MAHESHWARI, S N and PRASAD, R, Some new separation axioms, Ann. Soc. Scl. Bruxelles, T 3(89) (1975), 395-407, MR, 52\#6660

[14] MIODUSZEWSKI, J and RUDOLE, L , $H$-closed and extremely disconnected spaces, Dissertations Math. 66 (1969).

[15] REILLY, I L. and VAMANAMURTHY, M K, On spaces in which every denumerable subspaces is discrete, Math. Vesnik 38 (1986), 97-102

[16] THOMPSON, T, S-closed spaces, Proc. Amer. Math. Soc. 60 (1976), 335-338

[17] YOUNG, S P , Local discrete extensions of topologies, Kyungpook math. J. 11 (1977), 21-24 


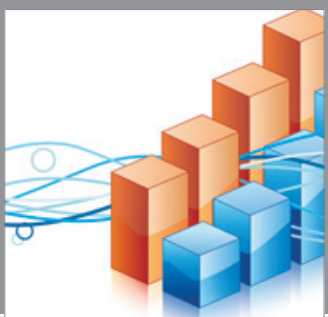

Advances in

Operations Research

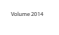

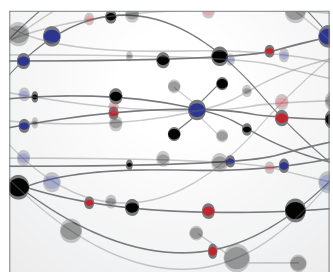

\section{The Scientific} World Journal
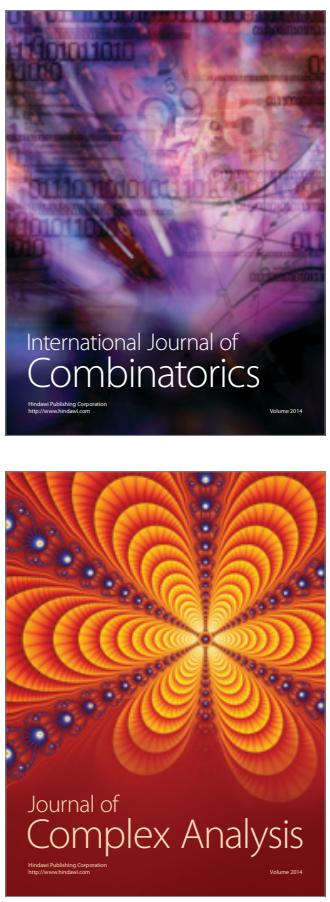

International Journal of

Mathematics and

Mathematical

Sciences
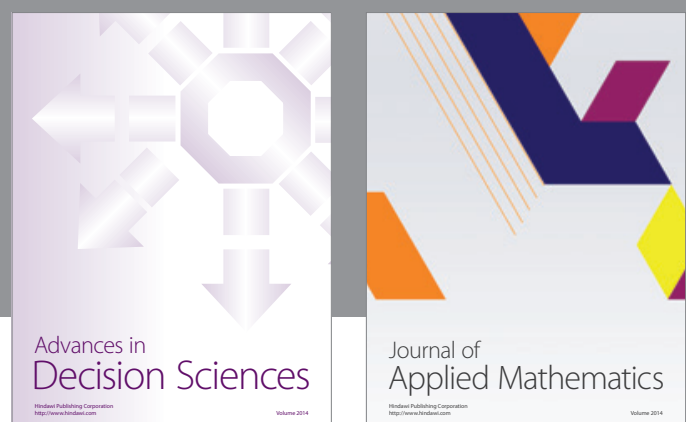

Journal of

Applied Mathematics
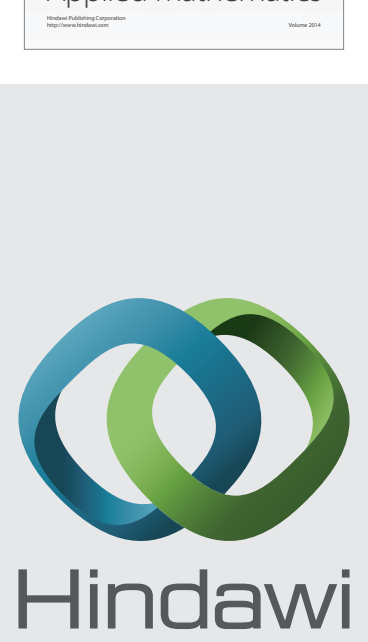

Submit your manuscripts at http://www.hindawi.com
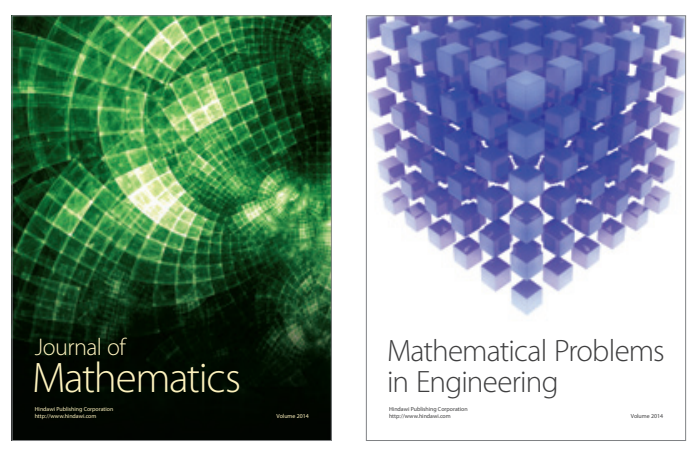

Mathematical Problems in Engineering
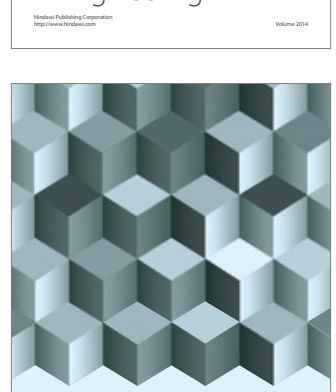

Journal of

Function Spaces
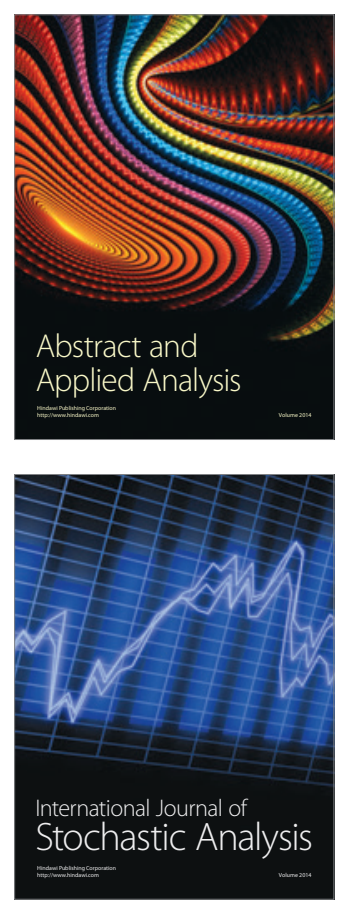

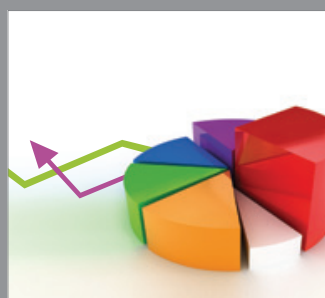

ournal of

Probability and Statistics

Promensencen
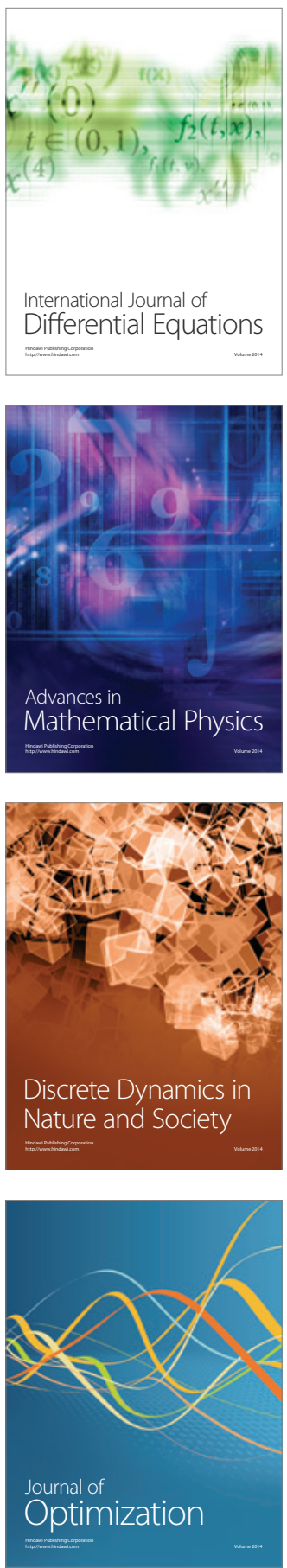